\title{
CONVERGENCE OF APPROXIMATING POLYNOMIALS
}

\author{
PHILIP C. CURTIS, JR. ${ }^{1}$
}

I. The problem we wish to consider is the following. For each positive integer $n$, let $E_{n}$ be a finite subset of $[-1,1]$ containing at least $n$ points. For a real-valued continuous function $f$ defined on $[-1,1]$ let $p_{n}\left(f, E_{n}\right)$ be the unique polynomial of degree at most $n-1$ of best approximation in the Chebycheff sense to $f$ on $E_{n}$. Is it possible to choose a fixed sequence $\left\{E_{n}\right\}$ so that for each $f$, continuous on $[-1,1], p_{n}\left(f, E_{n}\right)$ converges to $f$ uniformly on $[-1,1]$ ?

A classical result of Faber [4] states that if, for each $n, E_{n}$ contains exactly $n$ points, this choice is never possible. In this case, of course, $p_{n}\left(f, E_{n}\right)$ is just the polynomial which interpolates to $f$ at the points of $E_{n}$.

In this paper we shall prove that the result of Faber still holds if each $E_{n}$ contains no more than $n+1$ points. On the other hand, letting $\|f\|=\sup _{-1 \leqq t \leqq 1}|f(t)|$, we obtain $\left\|f-p_{n}\left(f, E_{n}\right)\right\| \rightarrow 0$ for each $f$ continuous on $[-1,1]$, if and only if there exists a constant $K$ independent of $n$, such that for each polynomial $p_{n}(x)$ of degree at most $n-1$, if $\left|p_{n}(x)\right| \leqq 1$ for each $x \in E_{n}$, then $\left\|p_{n}\right\| \leqq K$.

The existence of such sets $E_{n}$ was first proved by Bernstein [1, pp. 55-57]. In fact $E_{n}=\{\cos (k \pi / m)\}, k=0,1, \cdots, m$, where $m / n>\pi / 2 \cdot 2^{1 / 2}$ is a simple example. It is further shown in [1] that for each fixed $\lambda>1$ if $k_{n}$ satisfies $k_{n} / n>\lambda$ then we may choose a sequence $\left\{E_{n}\right\}$ with the desired properties and such that the cardinality of $E_{n}=k_{n}$. Namely, assuming $k_{n} \leqq 2 n$, let $E_{n}$ consist of the points $\cos ((2 k-1) / 2 n) \pi, k=1, \cdots, n$, together with the points $\cos (l t \pi / n)$ where $l$ is an integer satisfying $k_{n}-n-1=[n / l]$ and $t=0,1, \ldots$, $[n / l]$.

The author wishes to express appreciation for valuable conversations with W. Cheney and Y. Katznelson on these matters.

II. Let $C=C[-1,1]$, the Banach space of real-valued continuous functions on $[-1,1]$ provided with the norm $\|f\|=\sup _{-1 \leq t \leq 1}|f(t)|$. Let $H_{n}$ be the $n$ dimensional sub-space of polynomials of degree $n-1$. Denote by $P_{n}$ the mapping $f \rightarrow p_{n}\left(f, E_{n}\right) . P_{n}$ is a continuous mapping of $C$ onto $H_{n}$ satisfying $P_{m} P_{n}=P_{n}$ for $m \geqq n$. In general, $P_{n}$ is not linear, but if $E_{n}$ contains either $n$ or $n+1$ points, then $P_{n}$ is linear which is the crucial fact needed in the following:

Received by the editors December 23, 1960 and, in revised form, April 24, 1961.

${ }_{1}^{1}$ This research was done at the Space Technology Laboratories, Los Angeles and was supported by the United States Air Force. 
THEOREM 1. For $n=1,2, \cdots f\left(x\right.$ a sequence of finite subsets $E_{n}$ of $[-1,1]$. If each $E_{n}$ contains either $n$ or $n+1$ points, then there exists an $f \in C$ to which $p_{n}\left(f, E_{n}\right)$ fails to converge uniformly on $[-1,1]$.

Proof. Assume for the moment that $P_{n}$ is linear for each $n$. Let $f \in H_{n}$. Then if $m>n, P_{m}(f)=f$ since $P_{n}$ maps $C$ onto $H_{n}$ and $P_{m} P_{n}$ $=P_{n}$. By the Weierstrass approximation theorem the polynomials are dense in $C$, hence we may infer from the principle of uniform boundedness [3, Theorem II.3.6] that $P_{n}(f) \rightarrow f$ for each $f \in C$ iff $\sup _{n}\left\|P_{n}\right\|$ $<\infty$, where $\left\|P_{n}\right\|=\sup _{\|f\| \leq 1}\left\|P_{n}(f)\right\|$. But by $[5$, Hilfssatz 3, p. 495] if $P_{n}$ is any bounded projection of $C$ onto $H_{n},\left\|P_{n}\right\| \geqq \ln (n-1) / 8 \pi^{1 / 2}$.

Now $P_{n}$ is clearly linear if $E_{n}$ contains exactly $n$ points. If $E_{n}$ contains $n+1$ points $x_{i},-1 \leqq x_{1}<x_{2}<\cdots<x_{n+1} \leqq 1$, let $q_{n+1}(x)$ $=\sum_{k=0}^{n} a_{k} x^{k}$ and $r_{n+1}(x)=\sum_{k=0}^{n} b_{k} x^{k}$ be the unique polynomials determined by the conditions $q_{n+1}\left(x_{i}\right)=(-1)^{i}, r_{n+1}\left(x_{i}\right)=f\left(x_{i}\right), i=1,2$, $\cdots, n+1$. It is easily seen by considering the determinants involved that $a_{n} \neq 0$. Therefore, let $p_{n}(x)=r_{n+1}(x)-\left(b_{n} / a_{n}\right) q_{n+1}(x)$. The mapping $f \rightarrow p_{n}$ is clearly linear, since $f \rightarrow r_{n+1}$ and $f \rightarrow b_{n}$ are both linear. But $f\left(x_{i}\right)-p_{n}\left(x_{i}\right)=\left(b_{n} / a_{n}\right)(-1)^{i}, i=1,2, \cdots, n+1$. Therefore, $p_{n}=p_{n}\left(f, E_{n}\right)$ by the classical result of de la Vallée Poussin [2] which completes the proof.

We note two facts. First, it may be easily verified that if $E_{n}$ contains more than $n+1$ points, $P_{n}$ is never linear. Secondly, if $q_{n}\left(f, E_{n}\right)$ denotes the polynomial of best approximation to $f$ on $E_{n}$ in the sense of least squares, then the same argument as above shows that if $\left\{E_{n}\right\}$ is any sequence of finite subsets of $[-1,1]$ containing at least $n$ points, then for some $f \in C, q_{n}\left(f, E_{n}\right)$ fails to converge uniformly to $f$. This follows since the mapping $f \rightarrow q_{n}\left(f, E_{n}\right)$ is always linear and idempotent.

III. We now prove the convergence criterion.

THEOREM 2. For each $n>0$ let $E_{n}$ be a finite subset of $[-1,1]$. Then $\left\|f-p_{n}\left(f, E_{n}\right)\right\| \rightarrow 0$ for each $f \in C$ iff there exists a constant $K$ such that if $p \in H_{n},|p(x)| \leqq 1, x \in E_{n}$, then $\|p\|<K$.

Proof. This is a theorem of uniform boundedness type, and although the operators $P_{n}$ are nonlinear the proof resembles that for the linear case.

With no loss in generality, we assume each $E_{n}$ contains at least $n+1$ points. For fixed $E_{n}$ and $p \in H_{n}$ let $\delta(p)=\sup _{x \in E_{n}}|f(x)-p(x)|$. Then by a well-known result of de la Vallée Poussin $[2] p_{n}=p_{n}\left(f, E_{n}\right)$ is characterized uniquely by the condition that there exist $n+1$ points $x_{i}$ in $E_{n}, x_{i} \leqq x_{i+1}$, for which either 


$$
f\left(x_{i}\right)-p_{n}\left(x_{i}\right)=(-1)^{i} \delta\left(p_{n}\right), \quad i=1,2, \cdots, n+1,
$$

or

$$
f\left(x_{i}\right)-p_{n}\left(x_{i}\right)=(-1)^{i+1} \delta\left(p_{n}\right), \quad i=1,2, \cdots, n+1 .
$$

From this it follows easily that the operator $P_{n}$ is homogeneous, and if $q$ is a polynomial of degree $<n$, then for each $f \in C, P_{n}(f+q)$ $=P_{n}(f)+P_{n}(q)=P_{n}(f)+q$. Moreover $E_{n}$ satisfies the condition of the theorem iff $\sup _{n}\left\|P_{n}\right\|<\infty$. For, by the above remarks, if $p \in H_{n}$ and $|p(x)| \leqq 1, x \in E_{n}$, then $p=P_{n}\left(f, E_{n}\right)$ for some $f$, $\|f\| \leqq 2$. Conversely, if $\|f\| \leqq 1$, then $\left|p_{n}(x)\right| \leqq 2$ for $x \in E_{n}$ for otherwise $p(x) \equiv 0$ would provide a better approximation on $E_{n}$. Therefore, suppose $\sup _{n}\left\|P_{n}\right\| \equiv K<\infty$. For each $\epsilon>0$ choose a polynomial $q$ such that $\|f-q\|<\epsilon$. If $n_{\epsilon}$ is the degree of $q$ and $n>n_{\epsilon}$, then

$$
\begin{aligned}
\left\|f-P_{n}(f)\right\| & \leqq\|f-q\|+\left\|q-P_{n}(f)\right\| \\
& =\|f-q\|+\left\|P_{n}(q-f)\right\| \leqq \epsilon(1+K) .
\end{aligned}
$$

Therefore, $\left\|f-P_{n}(f)\right\| \rightarrow 0$ and the condition is sufficient.

Conversely, suppose $\left\|f-P_{n}(f)\right\| \rightarrow 0$ for each $f \in C$. Since each $P_{n}$ is continuous, $S_{n, k} \equiv\left\{f \in C:\left\|P_{n}(f)\right\| \leqq k\right\}$ is a closed subset of $C$. Therefore, by the Baire category theorem, for some $k>0, S_{k} \equiv \bigcap_{n=1}^{\infty} S_{n, k}$ contains an open set. Consequently, there exists a polynomial $q(x)$ and a positive number $\delta$ such that if $\|f\|<\delta$, then $f+q \in S_{k}$. Hence, for $n>$ degree of $q$ and $\|f\|<\delta$,

$$
\left\|P_{n}(f)\right\| \leqq\left\|P_{n}(q)\right\|+\left\|P_{n}(f-q)\right\| \leqq\|q\|+k .
$$

Using this and the continuity of each $P_{n}$ it follows that

$$
\sup _{n}\left\|P_{n}(f)\right\|<\infty, \quad\|f\|<\delta
$$

and the theorem is proved.

\section{BIBLIOGRAPHY}

1. S. Bernstein, Sur une modification de la formule d'interpolation de Lagrange, Comm. Soc. Math. Kharkow Inst. Sci. Math. Ukraine (4) 5 (1932), 49-57.

2. C. J. de la Vallée Poussin, Lȩons sur l'approximation des fonctions d'une variable réelle, Gauthier-Villars, Paris, 1919.

3. N. Dunford and J. Schwartz, Linear operators, Interscience, New York, 1958.

4. G. Faber, Über die interpolatorische Darstellung stetiger Funktionen, Jber. Deutsch. Math. Verein. 23 (1914), 197-210.

5. I. P. Natanson, Konstruktive Funktionentheorie, Akademie-Verlag, Berlin, 1955.

University of California, Los Angeles 